\title{
ANISOTROPIC MESH REFINEMENT IN SHIP FLOW SIMULATION WITH FREE SURFACE
}

\author{
JEROEN WACKERS, GANBO DENG AND MICHEL VISONNEAU \\ Laboratoire de Mécanique des Fluides, Ecole Centrale de Nantes \\ CNRS-UMR 6598, 44321 Nantes Cedex 3, France \\ e-mail: Jeroen.Wackers@ec-nantes.fr, Ganbo.Deng@ec-nantes.fr, \\ Michel.Visonneau@ec-nantes.fr
}

Key words: Combined criteria, Anisotropic refinement, Free-surface waves

\begin{abstract}
For the simulation of water flow with waves, grid refinement must be anisotropic to limit the total grid size. For these flows, the grid has to be refined at the water surface, to resolve the conservation law which indicates the surface position, and below the surface to resolve the water flow. A combined criterion is presented, based on the free-surface position and on the Hessian of the pressure. It is shown that this criterion creates suitable grids for two- and three-dimensional flows.
\end{abstract}

\section{INTRODUCTION}

The simulation of water flows with a free water surface is inherently a multiphysics problem, as the surface deformation through waves interacts with the viscous, turbulent flow below the surface. In a hydrodynamics flow solver, a model for the surface position is added to the standard Navier-Stokes equations. Accurate simulation of water flow requires a good resolution of this additional model, as well as the flow equations, on the same grid. Thus, adaptive grid refinement applied to water flows must take into account both these models. And therefore, refinement criteria that are a combination of different sensors are essential.

An adaptive grid refinement method has been developed $[1,2]$ for ISIS-CFD, the unstructured Reynolds-averaged Navier-Stokes solver developed by the Numerical Modelling group of LMF. This commercialised flow solver is aimed at the simulation of realistic flow problems in all branches of marine hydrodynamics. The method is therefore developed to be general and flexible, featuring anisotropic refinement on unstructured hexahedral grids, derefinement of previous refinements to enable unsteady flow computation, and full parallelisation including integrated dynamic load balancing. The anisotropic refinement is metric-based. Thus, the refinement criteria are $3 \times 3$ symmetric tensors in each cell, which indicate the local desired cell size in all directions. This formulation allows the straight- 
forward implementation of highly differing refinement criteria. The refinement method has already been succesfully applied to different test cases in marine flow simulation [2].

The focus of this paper is on the development of refinement criteria for hydrodynamic flows in this metric-based context. We discuss which features are relevant as refinement criteria and how multiple refinement criteria can be combined into one. Section 2 introduces the flow solver and the meshes used, section 3 gives an overview of the anisotropic mesh refinement method. Then section 4 discusses the necessity of combined criteria for flows with a free water surface. Section 5 shows the construction of a criterion that combines directional refinement at the free water surface with a pressure Hessian criterion. Three test cases in section 6 indicate that the criterion generates effective meshes for twoand three-dimensional free surface flows.

\section{FINITE-VOLUME METHOD}

The grid refinement is applied to a finite-volume method on unstructured grids, with a surface-capturing discretisation of the water surface, as implemented in the flow solver ISIS-CFD developed by the Numerical Modelling Group at LMF. This section describes the finite-volume discretisation and the type of meshes used, concentrating on those aspects that are most important for grid refinement and the construction of refinement criteria. Full details of the discretisation can be found in [3].

The ISIS-CFD flow solver resolves the incompressible Unsteady Reynolds-Averaged Navier Stokes equations in a two-fluid formulation. Free-surface water flows are modelled with a convection equation for the volume fraction of water $c_{i}$. The flow equations are discretised in a finite-volume framework. Pressure-velocity coupling is obtained through a Rhie \& Chow SIMPLE-type method: in each time step, the velocity updates come from the momentum equations and the pressure is given by the mass conservation law, transformed into a pressure equation. As the volume fraction $c_{i}$ is discontinuous, its convection equation is discretised with compressive flux functions. The resolution of this equation in each time step is decoupled from the pressure and velocity updates.

The discretisation is face-based. While all unknown state variables are cell-centered, the systems of equations used in the implicit time stepping procedure are constructed face by face. Fluxes are computed in a loop over the faces and the contribution of each face is then added to the two cells next to the face. This technique poses no specific requirements on the topology of the cells. Therefore, the grids can be completely unstructured, cells with an arbitrary number of arbitrarily-shaped faces are accepted.

For this study, unstructured hexahedral meshes are used (see figure 1). These meshes are generated with the HEXPRESS grid generator from NUMECA International. They offer the flexibility of an unstructured grid, yet have large regions where the mesh is structured. Variations in mesh size are handled by having small cells laying next to larger cells, a situation called 'hanging nodes' by other authors. In ISIS-CFD, due to the facebased algorithm, these cells are treated in exactly the same way as all the others: the larger cells are simply seen as cells with more than 6 faces. 


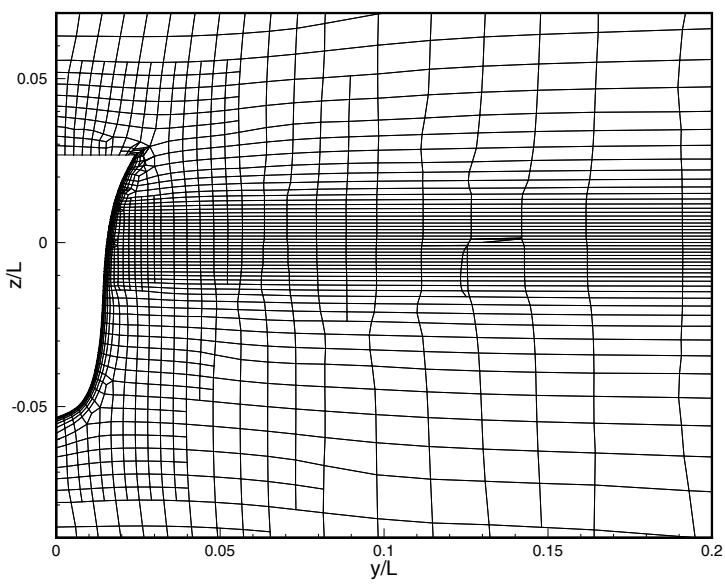

Figure 1: Cut through an unstructured hexahedral mesh.

Unstructure hexahedral grids are ideal for automatic grid refinement. Isotropic or anisotropic grid refinement can be applied to any of the hexahedral cells, the result will still be an unstructured hexahedral mesh. Therefore, locally refined meshes can be used directly in a flow solver that supports unstructured hexahedral meshes; no changes to the flow solver are needed to incorporate grid refinement.

\section{GRID REFINEMENT PROCEDURE}

The grid refinement procedure developed for ISIS-CFD [1, 2] is integrated completely in the flow solver. The method is entirely parallelised, including automatic redistribution of the grid over the processors. During a flow computation, the refinement procedure is called repeatedly. In such a call, first the refinement criterion is calculated, then in a separate step of the procedure the grid is refined based on this criterion. For steady flow, the refinement procedure converges: once the grid is correctly refined according to the criterion, further calls to the procedure no longer cause any changes.

\subsection{Anisotropic refinement}

Anisotropic refinement is essential for our type of grid refinement. Isotropic refinement is very costly in three dimensions, since each refinement means a division in eight (for a hexahedron). Thus, creating very fine cells to accurately resolve a local flow phenomenon becomes almost impossible. However, by applying anisotropic refinement for flow features that need a fine grid in only one direction (notably, the water surface!), the total number of cells required can be greatly reduced or much finer flow details can be resolved.

Also, in unstructured hexahedral original grids, cells of completely different aspect ratios lie side by side (see figure 1). Therefore, when refining, we need to control the size of the fine cells in all their directions independently, otherwise refined grids may have smoothly varying sizes in one direction, but repeated changes from fine to coarse and 
back to fine in another. Isotropic refinement is not enough to prevent this. Therefore, directional refinement is the mandatory choice.

\subsection{Tensor refinement criteria}

For directional refinement, a way is needed to specify different cell sizes in different directions. The use of metric tensors as refinement criteria is such a way. This technique was first developed for the generation and refinement of unstructured tetrahedral meshes $[4,5]$. It is also an extremely useful and flexible framework for the refinement of unstructured hexahedral meshes.

For tensor-based refinement, the refinement criterion in each cell is a $3 \times 3$ symmetric positive definite matrix $\mathcal{C}_{i}$. The refinement of the cells is decided as follows. Let the criterion tensors $\mathcal{C}_{i}$ in each cell be known (they are computed from the flow solution, see section 5$)$. In each hexahedral cell, the cell size vectors $\mathbf{d}_{j, i}(j=1, \ldots, 3)$, which are the vectors between the opposing face centres in the three cell directions, are determined. Next, the modified sizes are computed as:

$$
\tilde{\mathbf{d}}_{j, i}=\mathcal{C}_{i} \mathbf{d}_{j, i}
$$

Finally, a cell is refined in the direction $j$ when the modified size exceeds a given, constant threshold value $T_{r}$ :

$$
\left\|\tilde{\mathbf{d}}_{j, i}\right\| \geq T_{r} .
$$

The tensors $\mathcal{C}_{i}$ are direct specifications of the desired cell sizes: in the refined grid, the cell sizes are inversely proportional to the magnitude of the $\mathcal{C}_{i}$.

\section{THE NEED FOR COMBINED REFINEMENT CRITERIA}

Water waves are usually generated by the pressure and velocity disturbances created when the water flow passes around a foreign body, either a stationary object or a floating body such as a ship. These disturbances are not only generated at the surface, but also well below it; even a fully submerged object may create waves (see for example section 6.2 ). Once the waves are created, they propagate through orbital velocity fields in the water. Thus, to correctly resolve the generation and the propagation of a travelling surface wave, a good resolution is needed for the pressure and velocity fields below the surface.

Accurate resolution of the volume fraction equation is of prime importance as well. As the water surface is physically a discontinuity, the interface region for $c_{i}$ must be as sharp as possible. It can be shown [3], that a diffused interface increases numerical damping, so a too coarse grid at the surface will damp out waves. In our experience, the grid at the surface needs to be about twice as fine as the grid used in the vicinity of the surface.

Thus, to create suitable grids, a grid refinement criterion for water wave simulation must be based both on the pressure and velocity field and on the volume fraction. For these two, different indicators must be used. The reason for this, is that $c_{i}$ is discontinuous at the surface and constant everywhere else, while the pressure and the velocity are smooth 
in the whole flow field except at the surface. Therefore, gradient- or second-derivative based error indicators can be used to identify the regions of importance for the flow field below the surface, but not for $c_{i}$. They go to infinity at the surface, thus causing infinite refinement unless the criterion is artificially limited. Also, grid misalignment must be avoided in the surface region, as it leads to large errors in the volume fraction. Therefore, the refined grid must be as uniform as possible near the surface. Numerical evaluations of the derivatives of $c_{i}$ are never smooth, so they cannot guarantee uniform grids.

Therefore, a suitable refinement criterion for water flow with waves is an error indicator for the flow field and a simpler criterion for $c_{i}$, combined into one.

\section{PRESSURE HESSIAN - FREE SURFACE CRITERION}

A possible criterion for the simulation of flow with waves is based on the Hessian matrix of second derivatives of the pressure, combined with a criterion that refines in the normal direction of the surface for those cells where $c_{i}$ is neither 0 nor 1 .

\subsection{Free-surface criterion}

To resolve accurately the volume fraction, which is a discontinuity that is convected with the flow, it is sufficient to refine the grid in the direction normal to the water surface. When the surface is locally aligned with the cell directions, anisotropic refinement can be used to keep the total number of cells as low as possible.

The free-surface criterion is therefore based on a vector, normal to the surface, with length 1 . Thus, from equation (2) it follows that the threshold value $T_{r}$ directly indicates the desired cell size at the surface. The normal direction to the surface is computed from a $c_{i}$ field that is smoothed out by averaging over a cell and its neighbours, a given number of times. The gradient of this field gives the normal directions. The criterion vectors $\mathbf{v}_{i}$ are then chosen as the unit vectors in this normal direction for those cells where the smoothed $c_{i}$ field is non-zero, and as zero everywhere else. Switching based on the smoothed field guarantees that the mesh is refined also next to the surface, to create a margin of safety.

In tensor form, the free-surface criterion is implemented as matrices having only one non-zero eigenvalue, associated with the direction of the vector. In the directions normal to the vector, the eigenvalues are zero, so the desired grid size is infinity. Thus, the grid is only refined in the direction of the vectors. The tensors $\mathcal{C}_{S, i}$ are computed as follows $($ with $\otimes$ representing the tensor product):

$$
\mathcal{C}_{S, i}=\mathbf{v}_{i} \otimes \mathbf{v}_{i}
$$

The free-surface criterion has been used on its own in our earlier work [1, 2].

\subsection{Pressure Hessian}

Hessian-based criteria are often used to control anisotropic grid refinement [4]. We base this criterion on the pressure as this variable is insensitive to boundary layers, where 
we consider that the original unrefined grid should be sufficiently fine to ensure the best grid quality.

$$
\mathcal{H}_{i}=\left[\begin{array}{lll}
\left(p_{i}\right)_{x x} & \left(p_{i}\right)_{x y} & \left(p_{i}\right)_{x z} \\
\left(p_{i}\right)_{x y} & \left(p_{i}\right)_{y y} & \left(p_{i}\right)_{y z} \\
\left(p_{i}\right)_{x z} & \left(p_{i}\right)_{y z} & \left(p_{i}\right)_{z z}
\end{array}\right] .
$$

We compute the Hessian in each cell using a least-squares fit of a third-order polynomial to the solution in the cell, its neighbour cells and its neighbours' neighbours. There is no better third-order polynomial fit to these points, so the fit is fourth-order accurate. Therefore, its second derivatives are second order accurate, independent of the configuration of the neighbour cells. This is very important on refined meshes.

To compute the refinement criterion, the Hessian is modified with a power law:

$$
\mathcal{C}_{H, i}=\left(\mathcal{H}_{i}\right)^{p},
$$

where $\left(\mathcal{H}_{i}\right)^{p}$ has the same eigenvectors as $\mathcal{H}_{i}$ and eigenvalues that are those of $\mathcal{H}_{i}$ (in absolute value) to the power $p$. In general, we use $p=\frac{1}{2}$.

\subsection{The combined criterion}

For the final criterion, the two criteria above are combined. Even if the current implementation of the Hessian criterion has a behaviour similar to a free-surface criterion at the water surface, the real free-surface criterion is used as well because it guarantees that the grid at the surface is absolutely regular and that a safety zone of refined cells is generated around the surface.

The criteria are combined into one tensor criterion by taking a weighted maximum of the two tensors. We want $T_{r}$ to indicate directly the desired cell size (as for the free-surface criterion), so we apply a weighting factor $c$ only to the Hessian criterion:

$$
\mathcal{C}_{C, i}=\max \left(\mathcal{C}_{S, i}, c \mathcal{C}_{H, i}\right)
$$

The (approximate) maximum of the two tensors is computed using a procedure similar to the one in [5]. First, the eigenvalues and eigenvectors of the two tensors are computed. Then new eigenvalues are set for each tensor, as the maximum of the original eigenvalue and the length of the corresponding eigenvector when it is multiplied by the other tensor. This gives two approximations to the maximum tensor; the final tensor in each cell is a weighted average of these two.

\section{TEST CASES}

\subsection{Prism impacting on a water surface}

As a first test, we present the impact of a freely falling 2D prism on a water surface. This test is based on experiments by Peterson et al. [6]. In the present context, the interest of the case is that the $2 \mathrm{D}$ nature allows us to see in detail the functioning of the 
directional refinement. The prism for this test has a bottom angle of $20^{\circ}$, a width $W$ and an initial height $H$ both of $61 \mathrm{~cm}$, and a mass of $50 \mathrm{~kg} / \mathrm{m}$. The water has a density of $998.4 \mathrm{~kg} / \mathrm{m}^{3}$, the gravity is $9.81 \mathrm{~m} / \mathrm{s}^{2}$. Viscosity is neglected. The only movement allowed for the prism is vertical translation.

The numerical setup of the case is as follows. The free motion of the prism is computed using Newton's laws based on the integrated fluid forces on the prism, the motion is incorporated in the flow computation by block movement of the mesh. The same original mesh is used for all computations, this mesh has some local refinement around the prism. Refinement is called every 4 time steps. For this first test, the free surface criterion is used on its own; refinement is applied for $c_{i}$ between 0.3 and 0.9 to prevent perturbations from the tiny droplets in front of the main jet.

a)

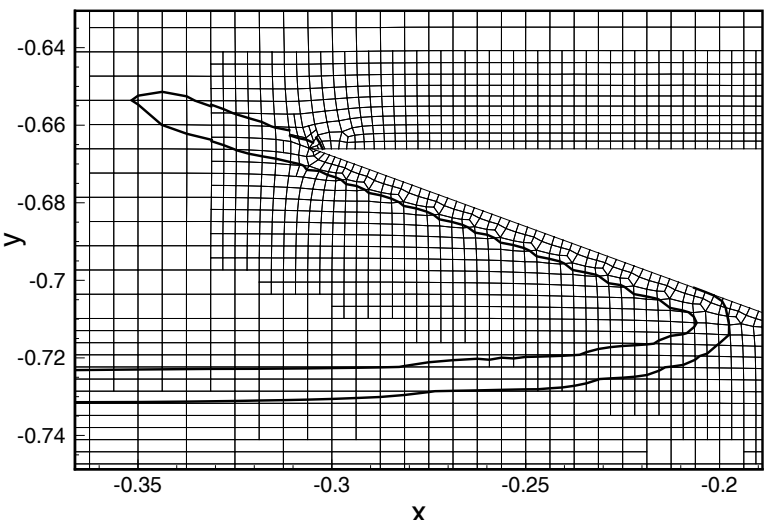

b)
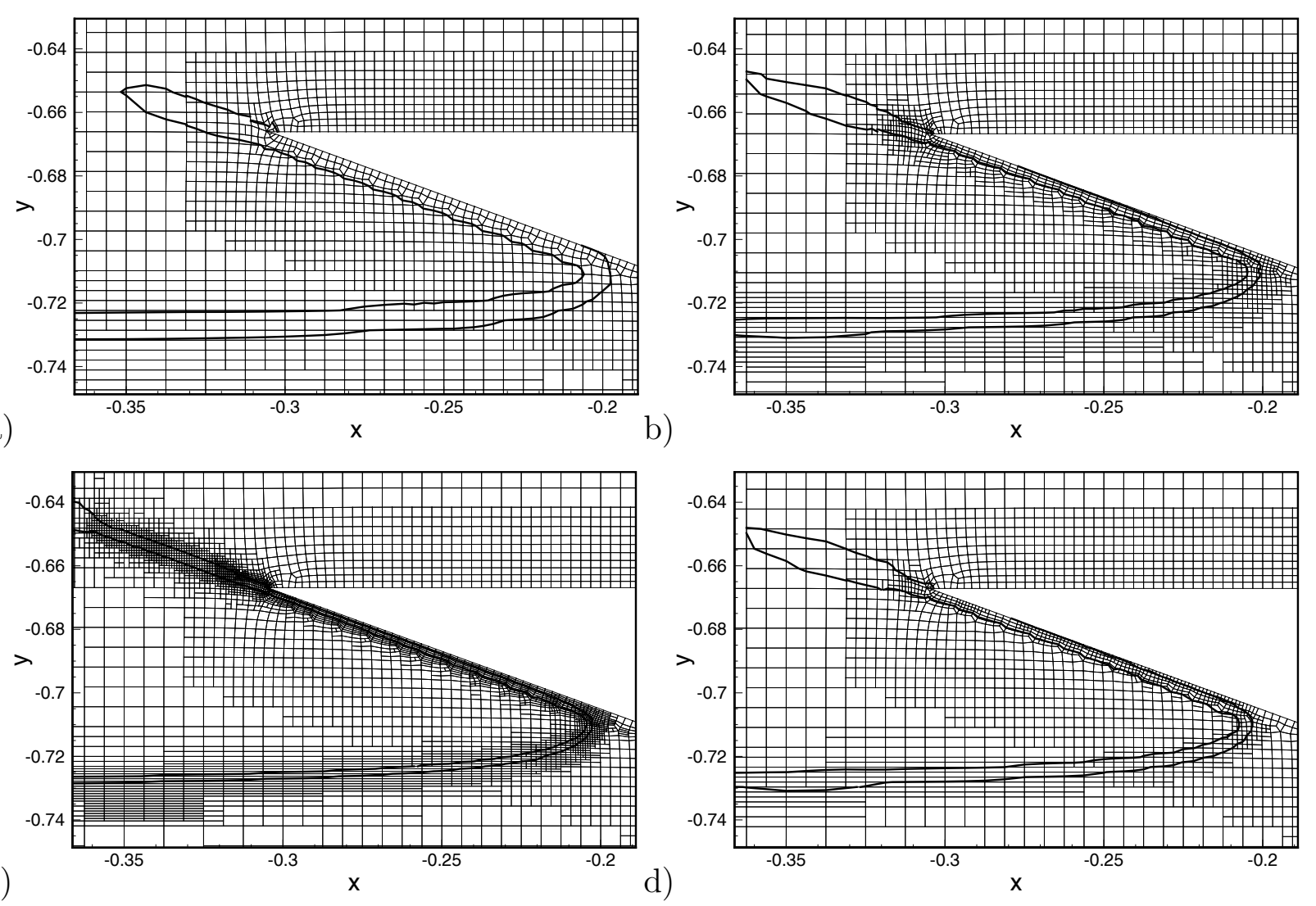

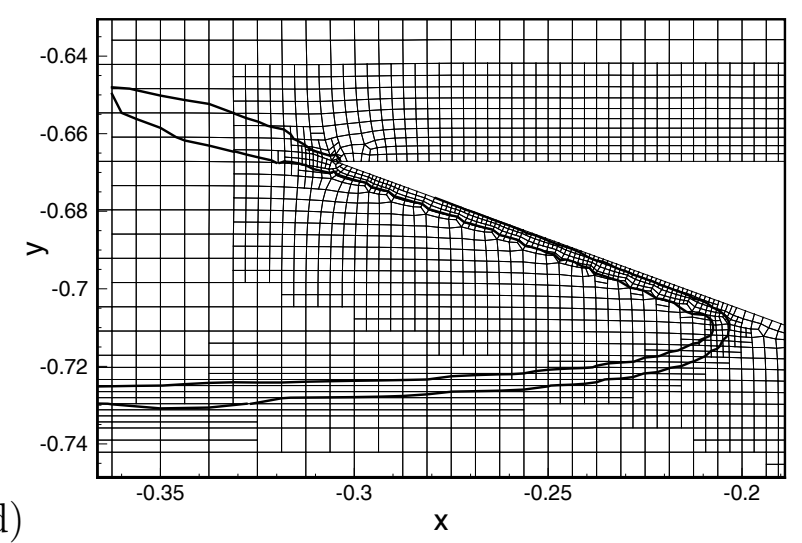

Figure 2: Mesh and $c_{i}=0.05$ and 0.95 isolines for the falling prism at $t=0.0175 \mathrm{~s}$ after initial impact. The figure is zoomed in on the left tip of the prism. The refinement threshold is $T_{r}=0.0033$ (a), $T_{r}=0.00165(\mathrm{~b})$, and $T_{r}=0.000825(\mathrm{c})$. Figure (d) represents the $T_{r}=0.00165$ case with convection buffer layers.

Figures $2 \mathrm{a}-\mathrm{c}$ show the mesh for three settings of the refinement threshold $T_{r}$ (equation (2)). The figures show how the criterion adapts the refined mesh both to the shape of the water surface and to the original mesh. Directional refinement is applied where possible, 
notably around the horizontal water surface and in the wall-aligned grid on the prism. Cells in the fine part of the original grid are not refined as often as cells further from the prism, so that the grid around the surface has a uniform size (figure 2a is an extreme example, where the finest part of the mesh is not refined at all). Note that the mesh is not refined around the tip of the water jet, due to the limitation to $c_{i}>0.3$.
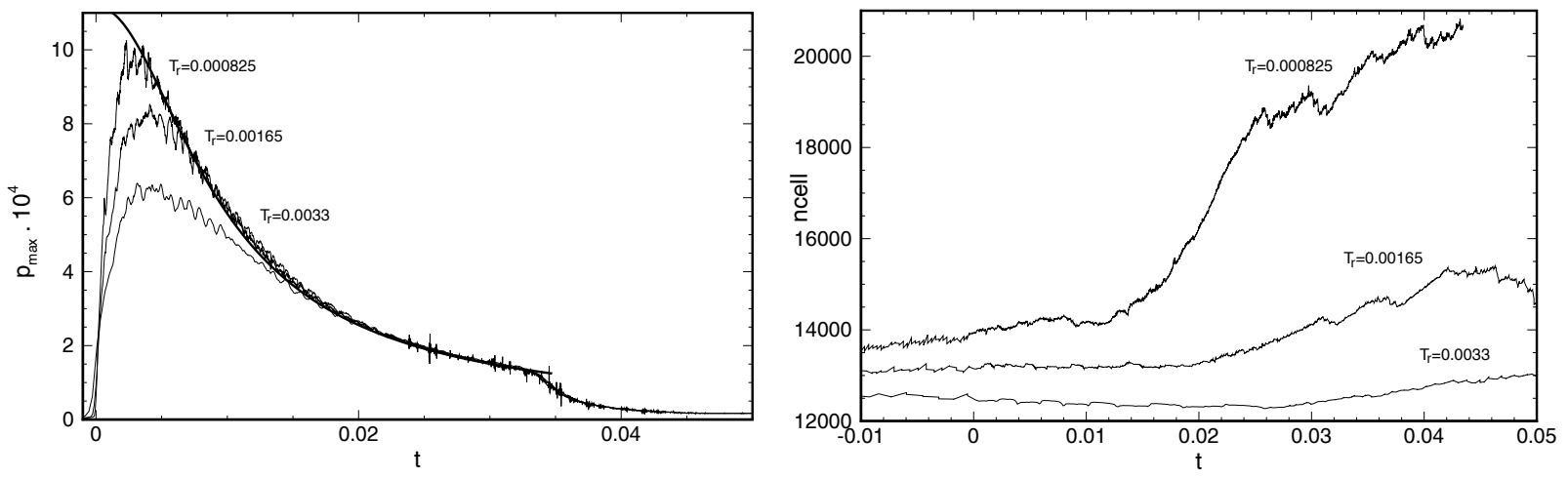

Figure 3: Pressure peak (left) and total number of cells (right) as a function of the time after initial impact, for the computations at three settings of the refinement criterion $T_{r}$, without convection buffer layers. The pressure peak is compared with the asymptotic solution of Scolan et al. [7].

The solution is characterised by a very strong pressure peak that moves outward over the prism during impact. As this peak is a highly localised feature, grid refinement is an effective way of resolving it well. Figure 3 shows the highest pressure on the prism as a function of time for the three simulations, compared with an asymptotic analytic solution by Scolan et al. [7]. As can be seen from this figure, the threshold has a very strong influence on the magnitude of the pressure peak; the agreement for the finest threshold is excellent. Also in figure 3, while the total number of cells increases with the reduction of the threshold, this increase is moderate, due mainly to the use of directional refinement.

\subsection{Immersed NACA0012 wing}

The second test case is meant to evaluate the behaviour of the combined refinement criterion for a two-dimensional wave field. We study the influence of the ratio parameter $c$ from equation 6 . The case is the geometry studied by Duncan [8], a wave train generated by an immersed NACA0012 profile of chord $0.203 \mathrm{~m}$ at 5 degrees angle of attack, with its centre point at $0.236 \mathrm{~m}$ below the surface. The inflow velocity is $0.8 \mathrm{~m} / \mathrm{s}$ and $R e=$ $1.42 \cdot 10^{5}$. The problem geometry can be found in figure 4 .

Four simulations are performed, starting from an original mesh that has some refinement around the profile but none at the surface. For each simulation, the grid is refined around the free surface to a target size $T_{r}=0.002 \mathrm{~m}$. Different values of $c$ give different sizes of the grid below the surface. The grid for $c=0.001$ is given in figure 4 , it shows refinement around the profile (notably at the leading edge), in a specific region between 


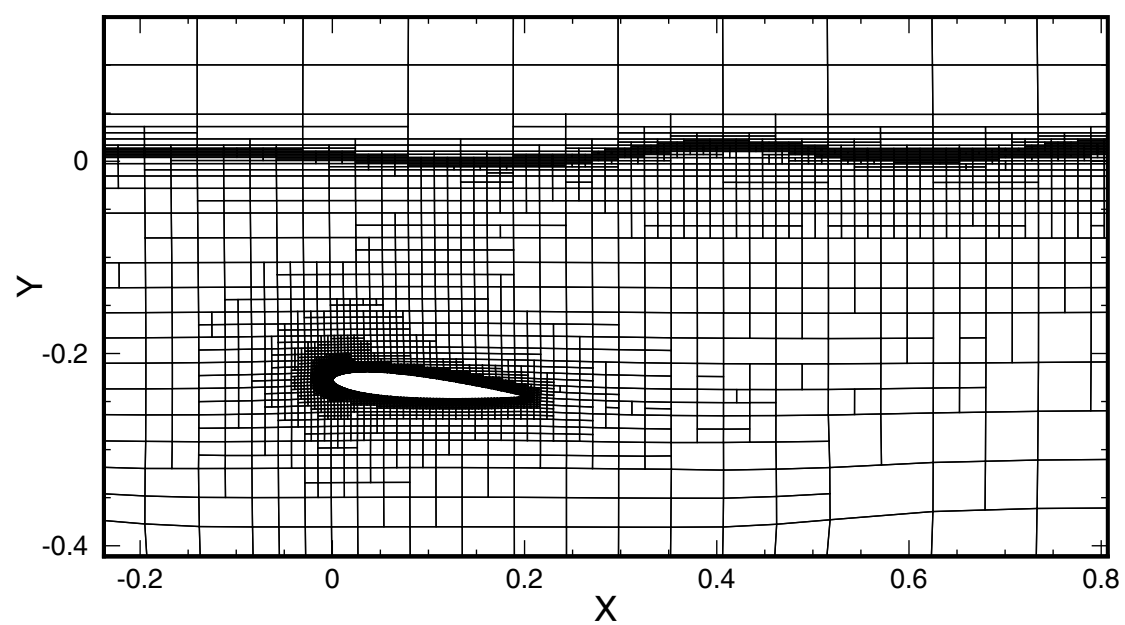

Figure 4: Refined mesh around the immersed profile for the Duncan case with $c=0.001$.

the profile and the surface, and in the wave field. A zoom of the meshes around the first wave is given in figure 5; the first figure is the result with refinement around the surface only, the last figure with $c=0.004$ has pressure-based refinement below the surface with the same size as the refinement specified by the free-surface criterion. Interestingly, while the refinement procedure is fully anisotropic, the pressure Hessian criterion creates only square cells below the waves.

Table 1: Number of cells in the refined meshes, Duncan test case.

\begin{tabular}{ccccc}
\hline$c$ & 0 & 0.001 & 0.002 & 0.004 \\
\hline cells & 3539 & 10244 & 37035 & 189860 \\
\hline
\end{tabular}

In figure 6 , the position of the free surface is given for the four cases. The results on the three grids produced with the combined criterion are very similar. Thus, it is not necessary to refine the grid below the surface to the same size as the grid at the surface, twice and even four times coarser cells are acceptable. As the total number of cells increases strongly with the parameter $c$ (table 1), this parameter should be kept low.

\subsection{Series 60 wave pattern}

The final test concerns the flow around a Series 60 ship in still water at Froude number $F r=0.316$ and $R e=5.3 \cdot 10^{6}$. The computation is started from a coarse mesh that has no initial refinement at all around the free surface, it is used to show that a sensible refined mesh for free-surface ship flow can be obtained entirely with automatic grid refinement. The grid is obtained with a target cell size $T_{r}=0.001 \mathrm{~L}$ and a ratio $c=0.004$, the original grid has $253 \mathrm{k}$ cells and the final grid $2.81 \mathrm{M}$ cells. 
a)

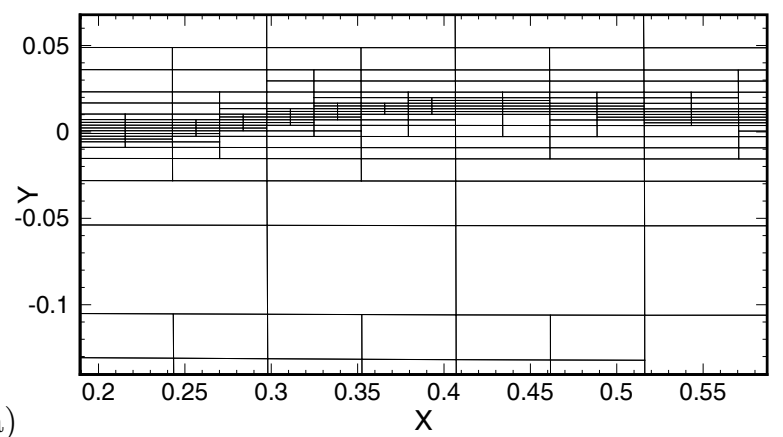

b)

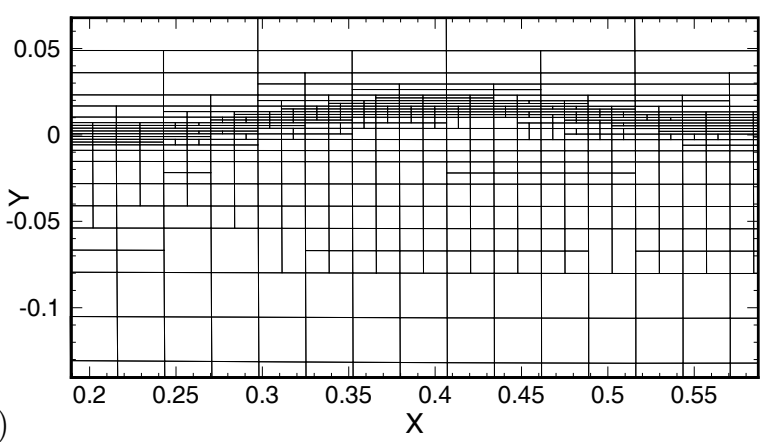

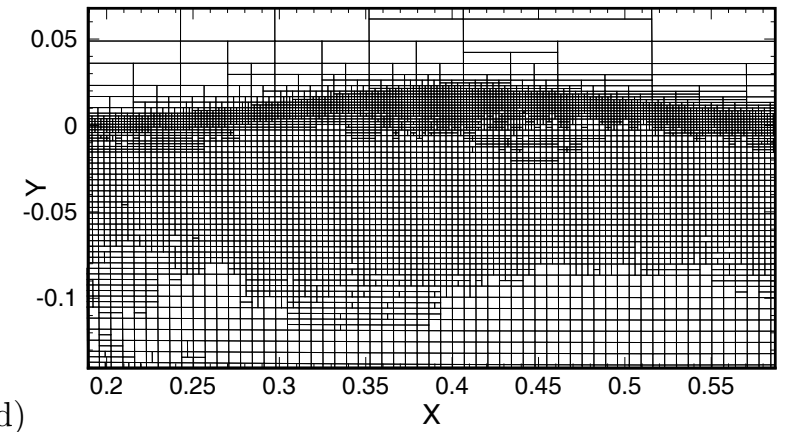

c)

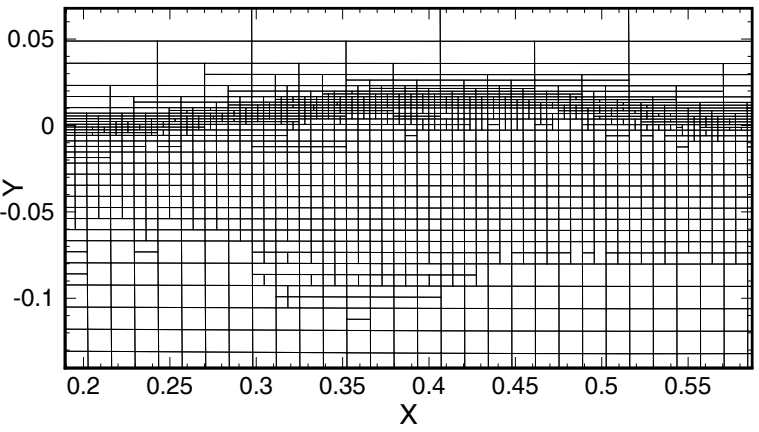

d)

Figure 5: Refined meshes at the first wave for the Duncan case with surface-only refinement $c=0.0$ (a), with $c=0.001(\mathrm{~b}), c=0.002(\mathrm{c})$, and $c=0.004(\mathrm{~d})$.

Four $X$ cross-sections of the refined mesh can be seen in figure 7 . The free-surface criterion applies directional refinement around the undisturbed surface; without this refinement, the interface would be dispersed in front of the ship, so accurate computations would be impossible. Refinement in all directions appears in the strongest waves. Pressure-based refinement is seen at the bow of the ship, below the hull at the stern, and below the waves. It is concentrated near the sharp peaks of the wave system. As for the Duncan test case, the refined cells below the waves are predominantly square.

The wave pattern, compared with experiments from IIHR [9], is given in figure 8. The correspondance is good, comparable with the results obtained in [3] on structured grids of $3.8 \mathrm{M}$ cells. Given the results of the Duncan test, a similar accuracy can probably be obtained with fewer cells if $c$ is reduced. This is a subject for further study.

\section{CONCLUSION}

It is shown that anisotropic grid refinement is essential for water flow with a free surface. Also, refinement criteria for these flows must refine both around the surface, to resolve the convection equation for the volume fraction, and in the region below it in order to capture the orbital flow fields. Due to the discontinuous solution at the surface, different criteria must be used to control the refinement in these two regions. Tests show, that a criterion which combines refinement normal to the surface with Hessian-based 


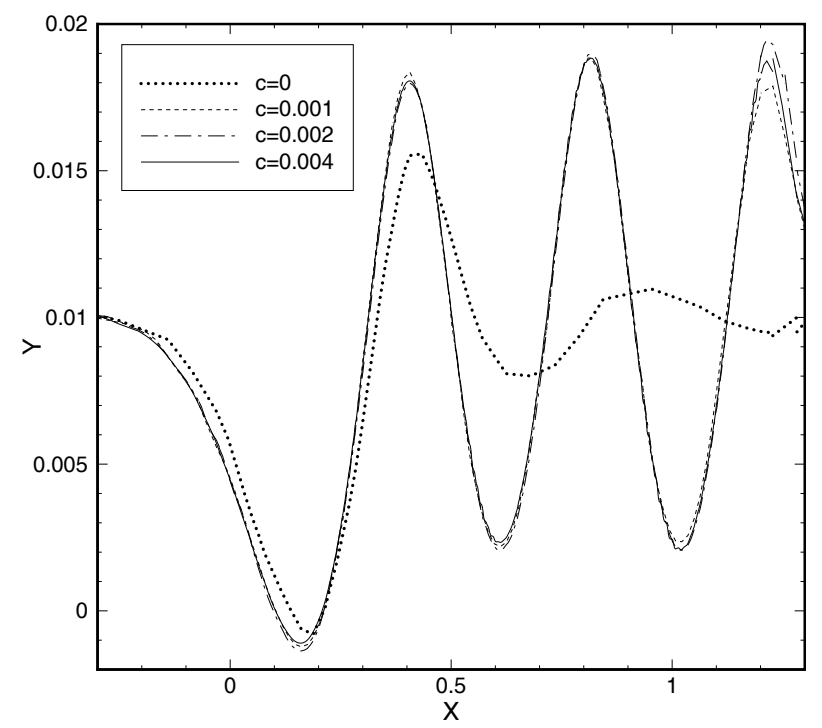

Figure 6: Duncan test case, free-surface positions of the four computations.

refinement can accurately resolve free-surface flows when starting from uniformly coarse original grids. Optimal results are obtained when the grid at the surface is two to four times finer than directly below it.

\section{REFERENCES}

[1] Wackers, J. and Visonneau, M. Adaptive grid refinement for ship flow computation. ADMOS 2009, Brussels, Belgium (2009).

[2] Wackers, J., Ait Said, K., Deng, G.B., Queutey, P., Visonneau, M., and Mizine, I. Adaptive grid refinement applied to RANS ship flow computation. 28th Symposium on Naval Hydrodynamics, Pasadena, California (2010).

[3] Queutey, P. and Visonneau, M. An interface capturing method for free-surface hydrodynamic flows. Computers \&f Fluids (2007) 36(9):1481-1510.

[4] Alauzet, F. and Loseille, A. High-order sonic boom modeling based on adaptive methods. J. Comp. Phys. (2010) 229(3):561-593.

[5] George, P.L. and Borouchaki, H. Delaunay Triangulation and Meshing: Application to Finite Elements, Hermes (1998).

[6] Peterson, R., Wyman, D., and Franck, C. Drop tests to support water-impact and planing boat dynamics theory, Coastal Systems Station, Panama City, USA (1999).

[7] Scolan, Y.-M., Coche, E., Coudray, T., and Fontaine, E. Etude analytique et numérique de l'impact hydrodynamique sur des carènes dissymétriques, 7es Journées de l'Hydrodynamique, Marseille, France (1999). 
a)

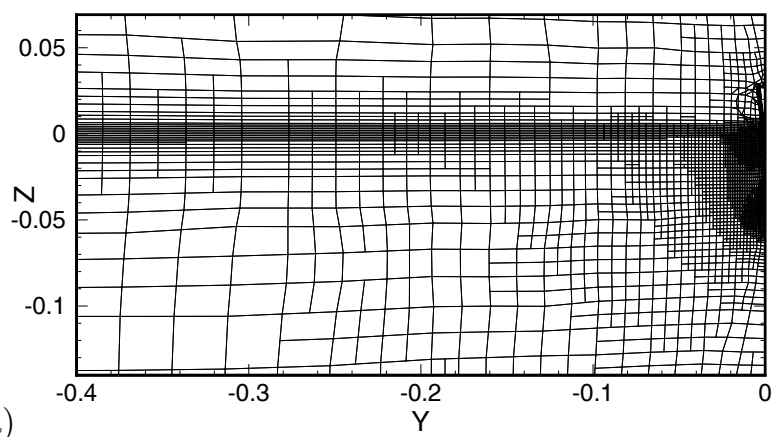

b)
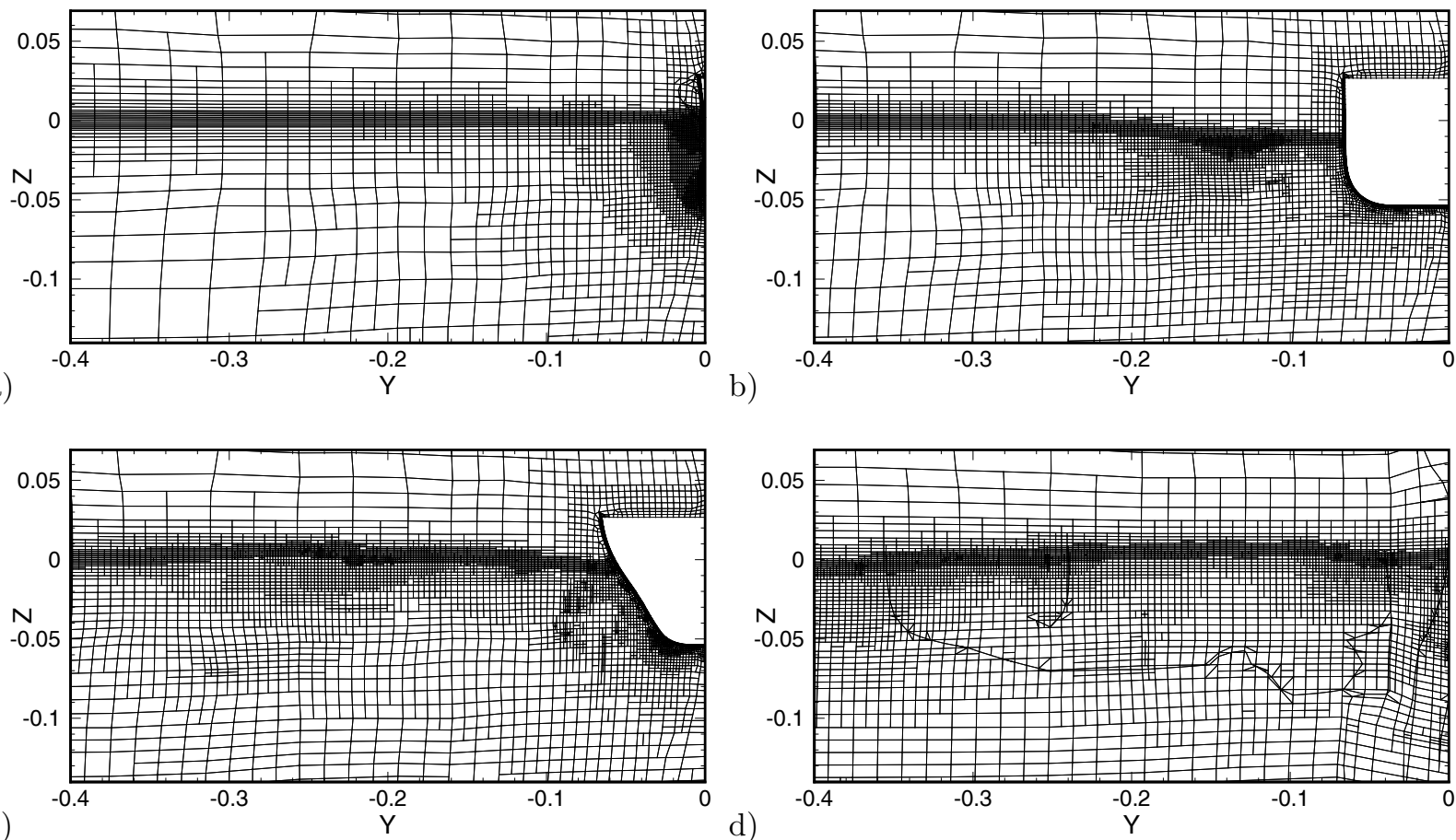

d)

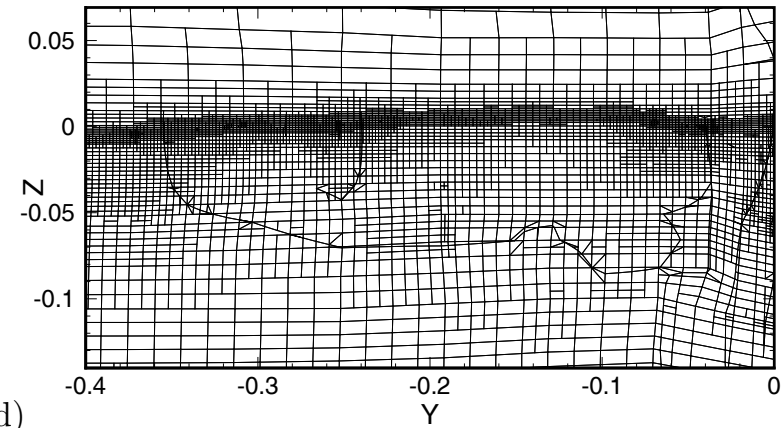

Figure 7: Series 60, cross-sections of the grid at $X / L=0.0$ (a), at $X / L=0.4$ (b), at $X / L=0.8$ (c) and behind the ship at $X / L=1.2(\mathrm{~d})$.

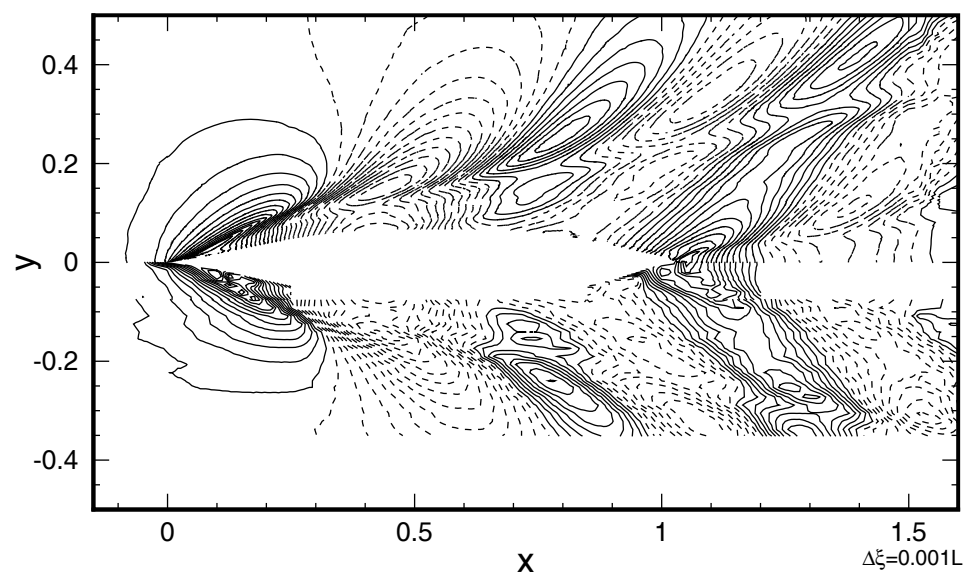

Figure 8: Wave pattern of Series 60, compared with experimental results from IIHR.

[8] Duncan, J.H. The breaking and non-breaking wave resistance of a two-dimensional hydrofoil. J. Fluid Mech. (1983) 126:507-520.

[9] Longo, J. and Stern, F. Effects of drift angle on model ship flow. Exp. Fluids (2002) 32:558-569. 